\title{
APLICAÇÃO DAS EQUAÇÕES DE ESTADO EM MISTURAS DE GASES REAIS NAS EMBALAGENS COM ATMOSFERA MODIFICADA
}

César Augusto CANCIAM*

\author{
*Mestre em Engenharia Química, professor lotado no Departamento Acadêmico de Engenharia Química da \\ Universidade Tecnológica Federal do Paraná - Câmpus Ponta Grossa, canciam@utfpr.edu.br
}

Recebido em: 04/09/2013 - Aprovado em: 20/12/2013 - Disponibilizado em: 15/01/2014

\begin{abstract}
RESUMO: O conhecimento do volume específico de uma mistura gasosa em embalagens com atmosfera modificada permite determinar o volume do espaço-livre, a massa da mistura gasosa utilizada e o volume do produto na embalagem. O objetivo deste trabalho foi utilizar as equações de estado para mistura de gases reais de Van der Waals e de Redlich-Kwong na determinação do volume específico dessa mistura. Para tanto, foi considerada, como exemplo, uma mistura de gases, em condições de temperatura e pressão interna pré-definidas. Os valores obtidos para o volume específico nesse exemplo foram próximos, indicando que as referidas equações de estado são satisfatórias.
\end{abstract}

PALAVRAS-CHAVE: Equação de estado. Embalagem. Alimento. Atmosfera modificada.

\section{APPLICATION OF THE STATE EQUATIONS IN MIXTURE REAL GASES IN MODIFIED ATMOSPHERE PACKINGS}

\begin{abstract}
The knowledge of the specific volume of a gaseous mixture in modified atmosphere packings allows to determine the head space, the mass of the used gaseous mixture and the volume of the product in the packing. The objective of this work was to use the state equations for mixture of real gases of Van der Waals and of Redlich-Kwong in the determination of the specific volume of that mixture. For so much, it was considered, as example, a mixture of gases, with temperature and pressure intern defined. The values obtained for the specific volume in that example, they were close, indicating that the state equations are satisfactory.
\end{abstract}

KEYWORDS: State equation. Packing. Food. Modified atmosphere.

\section{INTRODUÇÃO}

Existe uma forte tendência em modificar os meios de conservação de alimentos, de maneira a manter os alimentos mais frescos, conservando a aparência visual e o valor nutritivo por um período de tempo mais prolongado. A atmosfera modificada é uma tecnologia de conservação de alimentos que atende à crescente demanda dos consumidores por alimentos mais frescos e com maior vida útil (BEIRÃO, 2000).
A tecnologia de embalagem com atmosfera modificada consiste na substituição parcial ou total do ar em contato com o alimento por uma mistura gasosa de composição conhecida (POLETO, 2000).

Essa tecnologia começou ainda no final do século XIX, em que carnes frescas foram conservadas durante 4 a 5 semanas em atmosferas modificadas com elevado teor de gás carbônico (SARANTÓPOULOS et al., 1998).

A utilização de embalagens com atmosfera modificada para alimentos 
apresenta como vantagens: o aumento da vida útil; a redução de perdas de distribuição; a eliminação ou redução de conservantes; a possibilidade da comercialização de produtos alimentícios de alta qualidade (cor, aroma e frescor conservados); melhor apresentação do produto e a opção para implantação de centrais de acondicionamento com linhas automáticas para grandes volumes de produção; entre outras vantagens. Contudo, a utilização de embalagens com atmosfera modificada apresenta custo adicional com embalagem, equipamentos e gases; a técnica não é universalmente efetiva e exige uma otimização; necessidade de controle de temperatura durante o acondicionamento, distribuição, estocagem e venda; necessidade de controle de qualidade da matéria-prima e do acondicionamento do produto (SARANTÓPOULOS et al., 1998).

Os gases normalmente usados nas embalagens com atmosfera modificada são o oxigênio, o nitrogênio e o gás carbônico (SARANTÓPOULOS e SOLER, 1988).

O gás oxigênio é importante para a manutenção da coloração vermelha (no caso das carnes) e do metabolismo aeróbio (no caso de alimentos que respiram). Já o gás carbônico apresenta ação bacteriostática e fungistática sobre muitos tipos de microrganismos (agindo como um conservante). Enquanto que o gás nitrogênio inibe as reações de oxidação e o crescimento de fungos (no caso da exclusão de oxigênio).
O nitrogênio é um gás inerte e devido à sua baixa solubilidade e menor permeabilidade através da embalagem em comparação ao oxigênio e ao gás carbônico, é usado como gás de enchimento para evitar o desenvolvimento de vácuo na embalagem, enquanto os outros gases não inertes são consumidos do espaço-livre (SARANTÓPOULOS e SOLER, 1988, SARANTÓPOULOS et al., 1998).

Segundo Sarantópoulos e coautores (1998), cada produto alimentício requer uma combinação de gases adequada, aliada à natureza e qualidade inicial do produto, ao controle de temperatura, às propriedades de barreira da embalagem e a eficiência do equipamento de acondicionamento.

O conhecimento do volume específico da mistura gasosa permite determinar o volume do espaço-livre ou "head space", a massa da mistura gasosa utilizada e o volume do produto (SARANTÓPOULOS e SOLER, 1988, VAN WYLEN et al., 2009).

O objetivo deste trabalho foi aplicar as equações de estado de mistura de gases reais de Van der Waals e de Redlich-Kwong na determinação do volume específico da mistura gasosa utilizada nas embalagens com atmosfera modificada.

Para tanto, foi aplicada uma modelagem matemática em uma composição gasosa já avaliada experimentalmente. 


\section{MODELAGEM MATEMÁTICA}

$$
b=y_{i} \cdot b_{i}
$$

As variáveis $P, V$ e $T$ (pressão, volume e temperatura, respectivamente) descrevem o estado macroscópico de uma mistura em equilíbrio. Uma equação de estado é uma relação entre essas variáveis de estado macroscópico no estado de equilíbrio, de maneira que (SMITH et al., 2007):

$$
f(P, V, T)=0
$$

A equação de Van der Waals é classificada como uma equação de estado semiempírica e considera o efeito das forças atrativas existentes entre as moléculas dos gases constituintes da mistura, podendo ser escrita na forma de (MORAN e SHAPIRO, 2006):

$$
\left(P+\frac{a}{V_{e}^{2}}\right) \cdot\left(V_{e}-b\right)=R \cdot T
$$

Em que $V_{e}$ corresponde ao volume específico da mistura gasosa $\left(\mathrm{em} \mathrm{cm}^{3} / \mathrm{mol}\right)$, $R$ equivale à constante universal dos gases ideais $\left(R=82,05 \mathrm{~cm}^{3} \cdot\right.$ atm/mol.K); $a$ e $b$ correspondem às constantes determinadas pela Equação 3 e pela Equação 4, respectivamente (MORAN e SHAPIRO, 2006).

$$
a=\left(y_{i} \cdot \sqrt{a_{i}}\right)^{2}
$$

$$
\begin{aligned}
& a_{i}=\frac{27 \cdot R^{2} \cdot T_{c i}^{2}}{64 \cdot P_{c i}} \\
& b_{i}=\frac{R \cdot T_{c i}}{8 \cdot P_{c i}}
\end{aligned}
$$

Em que $T_{c i}$ corresponde à temperatura crítica do componente $i$ e $P_{c i}$, à pressão crítica desse componente.

Já a equação de Redlich-Kwong, muito usada na área de Engenharia, é dada pela Equação 8 (SMITH et al., 2007).

$$
P=\frac{R \cdot T}{V_{e}-b}-\frac{a}{\left[\left(V_{e} \cdot \sqrt{T}\right) \cdot\left(V_{e}+b\right)\right]}
$$


$\mathrm{Na}$ Equação 8, as constantes $a$ e $b$ são determinadas, respectivamente, através da Equação 3 e Equação 4.

A Equação 9 determina o valor de $a_{i}$ para a equação de Redlich-Kwong. Enquanto que a Equação 10 determina o valor de $b_{i}$ para esta mesma equação (MORAN e SHAPIRO, 2006).

$$
\begin{aligned}
& a_{i}=0,42748 \cdot\left(\frac{R^{2} \cdot T_{c i}^{5 / 2}}{P_{c i}}\right) \\
& b_{i}=0,08664 \cdot\left(\frac{R \cdot T_{c i}}{P_{c i}}\right)
\end{aligned}
$$

\section{APLICAÇÃO DA MODELAGEM MATEMÁTICA}

Como aplicação da modelagem matemática, será considerada a composição gasosa de $45 \%$ de gás oxigênio, $35 \%$ de gás nitrogênio e 20\% de gás carbônico (em volume). Esta mistura gasosa foi avaliada para carne fresca em porções, tendo este produto uma vida útil de 10 dias (SARANTÓPOULOS e SOLER, 1988).

Para efeito de cálculo, será considerada a temperatura desse produto constante e igual a $10^{\circ} \mathrm{C}$.

De acordo com Sarantópoulos e coautores (1998), a pressão interna de uma embalagem com atmosfera modificada deve estar entre 0,39 e 0,49 atm. Acima de 0,49 atm pode-se romper a termossoldagem da embalagem.

Para efeito de cálculo, será considerada uma pressão interna de 0,44 atm.

Com o objetivo de um melhor acompanhamento da aplicação da modelagem matemática, esta será dividida em subitens.

\section{- Cálculo da fração molar de cada} componente da mistura gasosa

De acordo com Van Wylen e coautores (2009), um gás real obedece à Equação 11:

$$
P \cdot V=Z \cdot n \cdot R \cdot T
$$

Em que $Z$ corresponde ao fator de compressibilidade do gás.

Considerando o volume do componente $i$ da mistura $\left(v_{i}\right)$, a Equação 11 pode ser reescrita na forma de (VAN WYLEN et al., 2009):

$$
P \cdot v_{i}=Z \cdot n_{i} \cdot R \cdot T
$$

$$
\text { Dessa forma a razão }\left(\frac{v_{i}}{V}\right)
$$

corresponde a:

$$
\left(\frac{v_{i}}{V}\right)=\left(\frac{n_{i}}{n}\right)
$$

Pela Equação 13, pode-se observar que a fração volumétrica do componente $i$ é 
igual à fração molar desse componente $\left(y_{i}\right)$, dada pela Equação 5 .

Assim, para uma composição gasosa de $45 \%$ de gás oxigênio, $35 \%$ de gás nitrogênio e 20\% de gás carbônico (em volume), a fração molar de cada componente equivale, respectivamente a, 0,$45 ; 0,35$ e 0,20 .

\section{- Cálculo das constantes $a$ e $b$ para} as equações de Van der Waals e de Redlich-Kwong

A Tabela 1 relaciona os valores para a temperatura crítica $\left(T_{c i}\right)$ e a pressão crítica $\left(P_{c i}\right)$ de cada componente.

Tabela 1 - Dados dos gases da mistura

\begin{tabular}{ccc}
\hline Gás & $P_{c i}(\mathrm{~atm})$ & $T_{c i}(\mathrm{~K})$ \\
\hline Oxigênio & 49,8 & 154,6 \\
Nitrogênio & 33,5 & 126,2 \\
Gás carbônico & 72,8 & 304,2
\end{tabular}

Fonte: SMITH et al. (2007).

Com base nos dados apresentados na Tabela 1, foram obtidos os valores de $a_{i}$ (Equação 6) e $b_{i}$ (Equação 7), considerando a equação de Van der Waals. A Tabela 2 relaciona estes valores encontrados.

Considerando a fração molar de cada componente e os dados indicados na Tabela 2 , os valores das constantes $a$ e $b$, para a equação de Van der Waals, correspondem, respectivamente a $1,7348.10^{6} \mathrm{~cm}^{6} \cdot$ atm $/ \mathrm{mol}^{2}$ e $36,4305 \mathrm{~cm}^{3} / \mathrm{mol}$.
Tabela 2 - Valores das constantes $a_{i}$ e $b_{i}$ na equação de Van der Waals

\begin{tabular}{ccc}
\hline Gás & $a_{i}\left(\mathrm{~cm}^{6} \cdot \mathrm{atm} / \mathrm{mol}^{2}\right)$ & $b_{i}\left(\mathrm{~cm}^{3} / \mathrm{mol}\right)$ \\
\hline Oxigênio & $1,3854 \cdot 10^{6}$ & 31,8397 \\
Nitrogênio & $1,3503 \cdot 10^{6}$ & 38,6370 \\
Gás carbônico & $3,6102.10^{6}$ & 42,8565 \\
\hline
\end{tabular}

Com base nos dados apresentados na Tabela 1, foram obtidos os valores de $a_{i}$ (Equação 9) e $b_{i}$ (Equação 10), considerando a equação de Redlich-Kwong. A Tabela 3 relaciona estes valores encontrados.

Tabela 3 - Valores das constantes $a_{i}$ e $b_{i}$ na equação de Redlich-Kwong

\begin{tabular}{ccc}
\hline Gás & $a_{i}\left(\mathrm{~cm}^{6} \cdot \mathrm{atm} \cdot \mathrm{K}^{1 / 2} / \mathrm{mol}^{2}\right)$ & $b_{i}\left(\mathrm{~cm}^{3} / \mathrm{mol}\right)$ \\
\hline Oxigênio & $1,7174 \cdot 10^{7}$ & 22,0687 \\
Nitrogênio & $1,5370 \cdot 10^{7}$ & 26,7801 \\
Gás carbônico & $6,3803 \cdot 10^{7}$ & 29,7047 \\
\hline
\end{tabular}

Considerando a fração molar de cada componente e os dados indicados na Tabela 3 , os valores das constantes $a$ e $b$, para a equação de Redlich-Kwong, correspondem, respectivamente a $2,3373.10^{7}$ $\mathrm{cm}^{6} \cdot$ atm. $\mathrm{K}^{2} / \mathrm{mol}^{2}$ e $25,2448 \mathrm{~cm}^{3} / \mathrm{mol}$.

\section{- Cálculo do volume específico da mistura gasosa a partir das equações de Van der Waals e de Redlich-Kwong}

Para uma pressão interna de 0,44 atm e uma temperatura constante de $10^{\circ} \mathrm{C}$ $(283,15 \mathrm{~K})$, a Equação 2 (equação de Van der Waals) pode ser reescrita na forma de: 


$$
\begin{aligned}
& \left(0,44 \cdot V_{e}^{3}-2,3248 \cdot 10^{4} \cdot V_{e}^{2}\right)+ \\
& \quad+\left(1,7348 \cdot 10^{2} \cdot V_{e}-6,32 \cdot 10^{3}\right)=0
\end{aligned}
$$

Nas mesmas condições, a Equação 8 (equação de Redlich-Kwong) pode ser reescrita na forma de:

$$
\begin{aligned}
& \left(7,4039 \cdot V_{e}^{3}-3,9093 \cdot 10^{5} \cdot V_{e}^{2}\right)+ \\
& \quad+\left(1,3029 \cdot 10^{7} \cdot V_{e}-5,9005 \cdot 10^{8}\right)=0
\end{aligned}
$$

Como se pode observar, as equações de Van der Waals (Equação 14) e de RedlichKwong (Equação 15) são equações cúbicas no volume específico, condizente com o que é comentado por Smith e coautores (2007).

As soluções da Equação 14 estão indicadas na Tabela 4. Enquanto que as soluções da Equação 15 estão indicadas na Tabela 5.

Tabela 4 - Valores do volume específico na equação de Van der Waals

\begin{tabular}{cc}
\hline Raiz da Equação & $V_{e}\left(\mathrm{~cm}^{3} / \mathrm{mol}\right)$ \\
\hline $1^{\text {a }}$ raiz & 52836,4 \\
$2^{\text {a }}$ raiz & $(0,0037+0,5207 . \mathrm{i})$ \\
$3^{\text {a }}$ raiz & $(0,0037-0,5207 . \mathrm{i})$ \\
\hline
\end{tabular}

Tabela 5 - Valores do volume específico na equação de Redlich-Kwong

\begin{tabular}{cc}
\hline Raiz da Equação & $V_{e}\left(\mathrm{~cm}^{3} / \mathrm{mol}\right)$ \\
\hline $1^{\text {a }}$ raiz & 52767,2 \\
$2^{\mathrm{a}}$ raiz & $(16,6603+35,1103 . \mathrm{i})$ \\
$3^{\mathrm{a}}$ raiz & $(16,6603-35,1103 . \mathrm{i})$ \\
\hline
\end{tabular}

Os valores indicados nas Tabelas 4 e 5 foram obtidos através de um software disponível na calculadora CASIO FX-850P Personal Computer.

\section{RESULTADOS E DISCUSSÃO}

Para uma mistura gasosa composta por $45 \%$ de gás oxigênio, $35 \%$ de gás nitrogênio e $20 \%$ de gás carbônico (em volume), a $10^{\circ} \mathrm{C}$ e pressão interna de 0,44 atm, o volume específico, considerando a equação de Van der Waals, corresponde a $52836,4 \mathrm{~cm}^{3} / \mathrm{mol}$ (aproximadamente 52,84 L/mol).

Nas mesmas condições, considerando a equação de Redlich-Kwong, o volume específico da mistura corresponde a 52767,2 $\mathrm{cm}^{3} / \mathrm{mol}$ (aproximadamente 52,77 L/mol).

Segundo Moran e Shapiro (2006), a equação de Redlich-Kwong é considerada melhor em relação à equação de Van der Waals, pois apresenta resultados mais próximos da realidade.

Embora o resultado obtido pela equação de Van der Waals seja considerado mais distante da realidade em relação ao obtido pela equação de Redlich-Kwong, a diferença entre eles $(0,07 \mathrm{~L} / \mathrm{mol})$ mostrou-se pequena.

O volume de espaço-livre ou "head space" corresponde ao espaço livre deixado entre o produto e a embalagem no processo de enchimento, e que no caso das embalagens com atmosfera modificada, é ocupado pela mistura gasosa (SARANTÓPOULOS e SOLER, 1988). 
De acordo com Van Wylen e coautores (2009), o produto do volume específico da mistura gasosa $\left(V_{e}\right)$ pelo número total de mols $(n)$ corresponde ao volume ocupado pela mistura $(V)$, que neste caso, corresponde ao volume do espaço-livre.

Dessa forma, conhecendo $V_{e}$ e $n$ utilizado no enchimento da embalagem, é possível determinar o volume do espaço-livre.

A Tabela 6 indica os possíveis valores para o volume de espaço-livre em função do número de mols da mistura gasosa.

Tabela 6 - Volume do espaço-livre em função do número de mols da mistura gasosa de acordo com a equação de Van der Waals

\begin{tabular}{cc}
\hline $\begin{array}{c}\text { Número total de mols da } \\
\text { mistura }\end{array}$ & $\begin{array}{c}\text { Volume do espaço-livre } \\
(\mathrm{L})\end{array}$ \\
\hline 1 & 52,84 \\
0,50 & 26,42 \\
0,02 & 1,06 \\
\hline
\end{tabular}

De maneira similar, a Tabela 7 indica os possíveis valores para o volume do espaço-livre em função do número de mols da mistura gasosa, considerando os resultados obtidos pela equação de RedlichKwong.

Através das Tabelas 6 e 7, pode-se observar a pequena diferença entre os resultados, indicando, que as equações de Van der Waals e de Redlich-Kwong são satisfatórias na determinação do volume específico da mistura gasosa empregada nas embalagens com atmosfera modificada.
Tabela 7 - Volume do espaço-livre em função do número de mols da mistura gasosa de acordo com a equação de Redlich-Kwong

\begin{tabular}{cc}
\hline $\begin{array}{c}\text { Número total de mols da } \\
\text { mistura }\end{array}$ & $\begin{array}{c}\text { Volume do espaço-livre } \\
(\mathrm{L})\end{array}$ \\
\hline 1 & 52,77 \\
0,50 & 26,39 \\
0,02 & 1,06 \\
\hline
\end{tabular}

A Tabela 8 relaciona o número de mols da mistura gasosa e a massa dessa mistura.

Para o cálculo da massa da mistura gasosa foi considerada a massa molar da mistura gasosa $(M M)$ equivalente a 33 g/mol. Este valor foi obtido através da Equação 16, sugerida por Covre (2001).

$$
M M=(0,45 \cdot 32)+(0,35 \cdot 28)+(0,20 \cdot 44)
$$

Na Equação 16, os valores 32 g/mol, $28 \mathrm{~g} / \mathrm{mol}$ e $44 \mathrm{~g} / \mathrm{mol}$ correspondem, respectivamente, às massas molares dos gases oxigênio, nitrogênio e gás carbônico. Os demais valores correspondem às frações molares de cada componente.

Tabela 8 - Massa da mistura gasosa em função do número de mols da mistura

\begin{tabular}{cc}
\hline $\begin{array}{c}\text { Número total de mols da } \\
\text { mistura }\end{array}$ & $\begin{array}{c}\text { Massa da mistura } \\
\text { gasosa }(\mathrm{g})\end{array}$ \\
\hline 1 & 33 \\
0,50 & 16,5 \\
0,02 & 0,66 \\
\hline
\end{tabular}

De acordo com Sarantópoulos e Soler, (1988), para produtos cárneos, o volume do espaço-livre e o volume do produto devem 
seguir uma proporção. Esta proporção varia de acordo com cada país. Por exemplo, na Dinamarca esta proporção é de 1:3, ou seja, 1 volume de espaço-livre para 3 volumes do produto. Outros países adotam a proporção de 1:1. Dessa forma, através do conhecimento do volume do espaço-livre consegue-se projetar o volume do produto na embalagem.

\section{CONCLUSÃO}

O conhecimento do volume específico de uma mistura gasosa permitiu determinar o volume do espaço-livre da embalagem e a massa da mistura gasosa utilizada.

Considerando uma mistura gasosa de $45 \%$ de gás oxigênio, $35 \%$ de gás nitrogênio e $20 \%$ de gás carbônico (em volume), a $10^{\circ} \mathrm{C}$ e pressão interna de 0,44 atm, as equações de Van der Waals e de Redlich-Kwong apresentaram resultados próximos.

\section{REFERÊNCIAS}

BEIRÃO, N. A. F. Tecnologia de atmosfera modificada. In: PIZZINATTO, A.; ORMENESE, R. C. S. C. Seminário de massas frescas e semiprontas, ITAL, Campinas, 2000, p. 91-95.

COVRE, G. J. Química Total. FTD, São Paulo, 2001. 664p.

MORAN, M. J.; SHAPIRO, H. N. Princípios de Termodinâmica para Engenharia. LTC, Rio de Janeiro, 2006. 681p.
POLETO, G. Equipamentos para Embalagem. In: PIZZINATTO, A.; ORMENESE, R. C. S. C. Seminário de massas frescas e semiprontas, ITAL, Campinas, 2000, p. 85-90.

SARANTÓPOULOS, C. I. G. L.; ALVES, R. M. V.; OLIVEIRA, L. M.; GOMES, T. C. Embalagens com atmosfera modificada. ITAL, Campinas, 1998. 114p.

SARANTÓPOULOS, C.; SOLER, R. M. Embalagens com atmosfera modificada/controlada. In: Novas tecnologias de acondicionamento de alimentos, ITAL/SBCTA, Campinas, 1988, p.105-114.

SMITH, J. M.; VAN NESS, H. C.; ABBOTT, M. M. Introdução à Termodinâmica da Engenharia Química. LTC, Rio de Janeiro, 2007. 638p.

VAN WYLEN, G. J.; SONNTAG, R. E.; BORGNAKKE, C. Fundamentos da Termodinâmica. Edgard Blücher, São Paulo, 2009. 659p. 